\title{
L'ORGANE AUDITIF DES ALCIOPIDES
}

\author{
PAR
}

Ed. BÉRANEGK

Avec la planche XVII

Les Annélides Polychètes sont celles dont l'organisation est la plus différenciée et dont les organes sensoriels sont les plus développés. La région céphalique de ces Vers porte des appendices tactiles et généralement des yeux variables en nombre et en complexité. Ce sont principalement ces deux types d'organes sensoriels qui permettent à ces Annélides d'explorer le milieu ambiant, et de s'en faire une certaine représentation en harmonie avec le degré de développement de leurs centres nerveux.

Les vésicules auditives ne font cependant pas défaut chez les Polychètes, mais leur présence n'a été signalée que dans quelques familles seulement. Ces vésicules ou otocystes sont annexées tantôt au segment céphalique, et les nerfs qui les desservent tirent leur origine de l'anneau péri-œsophagien (Arénicoles), tantôt au segment suivant ou premier segment troncal et sont alors innervées par le ganglion sous-œsophagien (Térébelles, Serpules).

JoURDAN ` a fait une étude histologique des otocystes des Arénicoles. Selon ce savant, les organes auditifs de ces Anné-

${ }^{1}$ Jourdan, Sur la structure des otocystes de l'Arenicola Grubii. Comptes Rendus Ac. Sc., t. 98.

Rev. SuISSE de Zool., T. I. 1893. 
lides sont situés dans la paroi dermo-musculaire même de la région céphalique et sont localisés exclusivement dans la couche musculaire de cette région. Ils ont une forme sphérique, sont entourés par une enveloppe conjonctive et appartiennent plutôt à la face dorsale de l'Arénicole. Ces organes auditifs constituent une sphère creuse dont la paroi est formée par une couche de cellules minces et allongées en fuseau. Le bord interne de ces cellules est limité par une cuticule portant probablement des cils vibratiles, leur bord externe s'effile et se résoud en un réseau fibrillaire en relation avec le nerf auditif. La cavité centrale des otocystes est remplie par un liquide albumineux dans lequel flottent des corpuscules calcaires sphériques, ou otolithes, variables en nombre et en dimension.

Les Alciopides qui sont des Annélides pélagiques possèdent, comme on le sait, deux yeux céphaliques volumineux. GREEFF, dans ses Untersuchungen über die Alciopiden, parues en 1876, leur attribue aussi des organes auditifs représentés par deux vésicules accolées chacune à la partie postérieure et inférieure d'un des globes oculaires. Ces vésicules, ainsi que KLEINENBERG $^{1}$ et moi ${ }^{2}$ l'avons montré, ne sont pas de nature sensorielle mais constituent des glandes qui font partie intégrante de l'œil. Il est facile de se rendre compte de la nature et de la fonction de ces pseudo-vésicules auditives, en les suivant dans les différentes phases larvaires que parcourent les Alciopides. On reconnait alors qu'elles sont comprises dans l'ébauche embryonnaire de l'œil de ces Annélides, et que leurs produits de sécrétion sont déversés dans la cavité optique, donnant ainsi naissance au corps vitré de l'adulte.

Puisque les otocystes décrits par GreefF ne sont que de

${ }^{1}$ Kleinenberg, Die Entstehung des Annelids aus der Larve von Lopadorynchus. Zeitschr. für wiss. Zool. 1886.

${ }^{2}$ Béraneck, Embryogénie et Histologie de l'ceil des Alciopides. Revue Suisse de Zoologie, 1893. 
simples glandes, s'ensuit-il que les Alciopides soient dépourvues d'organes auditifs? En étudiant l'embryogénie de l'œil de ces Vers, j’ai observé des organes spéciaux que l'on doit certainement assimiler à des otocystes et dont la description fera l'objet de ce travail.

Ces organes se développent relativement tard durant l'évolution embryonnaire. Ils deviennent surtout accusés chez les larves qui ont abandonné les Cténophores dans lesquelles elles habitaient, pour mener une vie pélagique indépendante. Ils ne sont pas transitoires mais se retrouvent, quoique légèrement modifiés, dans l'animal adulte.

Les organes auditifs des embryons d'Alciopes diffèrent entre autres par leur situation de ceux qui ont été observés chez quelques larves d'Annélides. Dans la Trochophore d'Eupomatus uncinatus par exemple, HatscheK ${ }^{1}$ signale l'existence de deux petites vésicules ectodermiques enfoncées dans le corps de la larve et renfermant des concrétions réfringentes ou otolithes. Chez les jeunes Alciopes, il n'en est pas de même: les organes auditifs ne sont pas inclus dans la paroi du corps, mais se présentent sous forme d'appendices de ce dernier. Ce sont de petits sacs plus ou moins ovoïdes rattachés à la larve par de courts pédoncules (fig. 8, $v$. au.); ils dépendent du premier segment troncal. Pour fixer les idées, je donne les dimensions d'un de ces organes auditifs appartenant à une larve déjà pélagique, organe dont je vais faire maintenant une description plus détaillée. Il mesure $350 \mu$ en longueur, $212 \mu$ en hauteur et $164 \mu$ en largeur; je crois pouvoir rapporter la larve dont il est tiré à l'Asterope candida.

Ces sacs auditifs, appendiculés au premier segment troncal, sont au nombre de deux et sont situés sur la ligne médio-latérale du corps. Ils ont la forme d'ovoïdes et sont creusés d'une spa-

' Hatscheк, Entwicklung der Trochophora von Eupomatus. Arb. Zool. Inst. Wien, 1886. 
cieuse cavité remplie par un milieu facilement coagulable. Ce milieu plasmique, ainsi que la cavité qui le renferme, se produit en partie aux dépens d'une dégénérescence de quelques-unes des cellules constituant le bourgeon ectodermique primitif plein dont dérive l'organe auditif de l'adulte (fig. $8, v$. au.). Il se constitue aussi par un processus identique à celui que j'ai signalé en décrivant le mode de formation des milieux dioptriques de l'œil des Alciopides. J'ai mentionné l'existence, dans la paroi du globe oculaire, de glandes unicellulaires déversant leurs produits de sécrétion dans la cavité optique et domnant naissance à des granules plus ou moins volumineux qui sont résorbés par la suite. Dans la paroi des sacs auditifs en voie de développement, j'ai aussi constaté la présence de glandes unicellulaires s'ouvrant dans la cavité centrale (fig. $2, c . g l$.). Ces glandes sont légèrement piriformes, elles mesurent environ $10 \mu$ en longueur et $8 \mu$ en largeur. Leur noyau, sphérique, a $6 \mu$ en diamètre et dans son réseau protoplasmique prolifèrent des granulations relativement volumineuses qui sont imprégnées d'une manière intense par les réactifs colorants. Le carmin boracique par exemple les colore en rouge foncé. Ces grains chromophiles (fig. 1 et $2, g r$.) ont en moyenne 2 à $3 \mu$ en diamètre; ils se déversent dans la cavité centrale de l'organe auditif et leur nombre ne fait qu'augmenter pendant toute la durée du développement larvaire des Alciopides.

Les granulations chromophiles des otocystes n'ont pas une existence transitoire comme c'est le cas pour les granulations correspondantes des yeux; elles persistent durant toute la vie de l'individu et flottent dans le milieu plasmique qui remplit la cavité de ces otocystes. Elles représentent des otolithes d'une nature très spéciale, puisqu'elles ne sont pas formées par des concrétions calcaires, mais qu'elles ont une origine glandulaire. De plus, ces granulations ne réfractent pas la lumière; elles sont très nombreuses, car sur une seule coupe de l'appendice auditif 
que je décris en ce moment, j'en ai compté environ deux cents.

Toutes ces granulations chromophiles ne proviennent pas, chacune séparément, des glandes unicellulaires dont j’ai parlé plus haut. Les premières granulations formées dans le réseau nucléaire de ces glandes et déversées dans la cavité de l'otocyste y mènent encore une vie indépendante et dans leur protoplasma on voit se dessiner d'autres granulations secondaires qui deviendront libres plus tard. Chez les larves d'Alciope, ces otolithes organiques sont groupés d'une manière assez uniforme dans la masse plasmique centrale de l'organe auditif. Il est assez intéressant de remarquer combien simples sont les procédés histogéniques à l'aide desquels se forment certains milieux des organes sensoriels des Alciopides. Les couchès dioptriques de l'œil, la masse plasmique centrale de l'otocyste, dérivent de glandes unicellulaires semblables, et les différences que l'on constate dans le sort ultérieur des produits d'activité de ces glandes sont uniquement dues aux exigences physiologiques spéciales des organes auxquels elles appartiennent.

Passons maintenant à la description de la paroi du sac auditif dont nous venons d'étudier le contenu. Chez la larve d'Astérope qui nous occupe, cette paroi mesure en moyenne $28 \mu$ d'épaisseur sur des coupes passant par le plan équatorial de l'organe. Elle a une structure relativement simple. Elle est constituée par des cellules ectodermiques qui tendent à se grouper en deux couches plus ou moins en continuité l'une avec l'autre: l'une externe, hypodermique; l'autre interne, sensorielle. L'organe auditif, dans son ensemble, est enveloppé par une mince cuticule identique à celle qui recouvre le corps de ces Annélides.

Dans la région équatoriale de l'otocyste, la couche hypodermique, celle dont dépend l'enveloppe cuticulaire, n'occupe guère que le quart de l'épaisseur totale de la paroi de l'organe. Les cellules dont elle est formée montrent un noyau généralement sphérique parsemé de fins granules chromophiles (fig. 2, c. hy.). 
Les contours de ces cellules ne sont pas nettement définis, de sorte que leur protoplasma tend à se fusionner en une couche continue et irrégulièrement réticulée. Les cellules qui constituent la couche sensorielle de l'otocyste sont allongées et disposées radiairement par rapport à la cavité centrale de cet organe. Flles possèdent un noyau elliptique orienté selon le grand axe de la cellule (fig. 1 et $2, c$. sens.). La membrane cellulaire est plus accusée que dans la couche précédente, de sorte que chaque élément conserve son individualité. Quelques-uns de ces éléments se transforment, comme je l'ai déjà mentionné, en glandes unicellulaires.

L'extrémité centrale des cellules de la couche sensorielle limite la cavité du sac auditif; leur extrémité périphérique plonge dans la couche protoplasmique réticulée externe, laquelle du reste n'est pas également développée en tous les points de la paroi de l'otocyste (fig. 1, c. sens.). Sur certaines coupes, les extrémités centrales des cellules acoustiques de l'organe auditif paraissent être bordées par une cuticule; à ce que j'ai pu observer, il s'agit ici plutôt d'une légère condensation du protoplasma cellulaire que d'une cuticule proprement dite. Je n'ai pas constaté d'une manière certaine la présence de cils vibratiles faisant saillie dans la cavité de l'otocyste, mais j'ai vu que le bord central des cellules envoie de courts prolongements protoplasmiques qui plongent dans le liquide albumineux tenant en suspension les granulations chromophiles décrites plus haut.

Les organes dont je viens d'indiquer les caractères histologiques ne peuvent être le siège, chez la larve d'Astérope, que du sens de l'audition ou du sens de l'équilibre. Quoiqu'ils diffèrent par leur situation et par certains détails de structure (présence de glandes unicellulaires, otolithes organiques et non calcaires) des otocystes des autres larves d'Annélides, l'ensemble de leur organisation en fait incontestablement soit des organes d'audition, soit des organes d'équilibration du corps. Il me reste éncore, 
pour compléter la preuve, à montrer les relations existant entre ces organes et la chaîne nerveuse ganglionnaire. La figure 1 est tirée d'une coupe passant par le pédicule qui rattache l'otocyste au premier segment troncal de l'Alciope. Ce pédicule est excentrique, c'est-à-dire qu'il ne correspond pas au plan équatorial de cet otocyste, mais part de la région postérieure de ce dernier. Ainsi que le démontre la série des coupes, le pédicule est formé aux dépens de la couche cellulaire externe de la vésicule auditive, couche qui prend dans cette région une grande extension et dont le revêtement cuticulaire vient se souder à celui du segment troncal correspondant (fig. 1, c.hy.).

Cette couche est encore parsemée de quelques noyaux plus ou moins sphériques, ponctués de nombreuses et fines granulations chromophiles. Elle constitue une masse protoplasmique réticulée, d'apparence conjonctive, entourant les cellules internes radiaires de l'organe auditif. Celles-ci sont beaucoup plus nettement séparées du reticulum protoplasmique externe que dans la région équatoriale de l'otocyste (comparer fig. 1 et 2, c.hy., c. sens.). Les cellules internes représentent seules des éléments sensoriels; elles sont innervées par le ganglion sous-œsophagien. Dans la fig. 1, on voit le nerf auditif (n.au.) qui se détache de ce ganglion, traverse le reticulum protoplasmique externe et se divise en deux rameaux enveloppant l'extrémité périphérique des celulles sensorielles. De ces rameaux se détachent de fines fibrilles qui se mettent en relation avec ces cellules et se perdent dans leur protoplasma. Dans la région équatoriale de l'otocyste, la mince couche protoplasmique réticulée externe me parait surtout servir de soutien aux fibres nerveuses qui se rendent aux éléments sensoriels bordant la cavité centrale de cet organe.

La description que je viens de donner se rapporte à une larve d'Alciope menant déjà une existence pélagique. Si l'on s'adresse à des Alciopides adultes, pour étudier la structure de leurs vésicules auditives, on éprouve parfois quelques diffi- 
cultés à retrouver, dans les organes complètement développés, les caractères sensoriels tellement accusés des organes correspondants de la larve. Ici encore, pour bien comprendre la morphologie de l'animal adulte, il faut recourir aux données fournies par l'évolution embryonnaire.

La constitution des sacs auditifs des Alciopides est sujette à varier, dans les détails, d'une espèce à l'autre. Je commencerai par décrire les organes qui s'écartent le moins du type larvaire, puis j'indiquerai une curieuse variation de ce type que l'on rencontre chez Alciope contrainii.

Les figures 3, 4 et 5 sont tirées de l'Asterope candida. Les sacs auditifs sont appendiculés au premier segment troncal; ils sont piriformes, et leur grand axe est perpendiculaire au plan de symétrie du corps. La cavité centrale n'est pas aussi marquée que chez la larve. Elle est relativement moins spacieuse, et sa forme peut devenir très irrégulière suivant les régions par lesquelles les coupes sont menées. Cette cavité est remplie par une masse plasmique plus dense que durant la période embryonnaire, ce qui est dû, principalement, à la dégénérescence d'une partie des grains chromophiles mentionnés plus haut. La masse plasmique constitue un pseudo tissu sous lequel sont encore englobées de nombreuses granulations chromophiles, les unes sphériques, les autres ovoüdes mesurant en moyenne $2 \mu$ en diamètre. Ainsi que je l'ai montré, ces granulations fonctionnent comme de véritables otolithes; elles ne sont biréfringentes ni dans la larve, ni dans l'adulte. Elles sont accumulées en certains points, plus espacées en d'autres; ainsi dans la figure 3, elles sont surtout réparties vers le bord interne de l'organe auditif. Parfois, la paroi de cet organe se renfle et forme une sorte de bourgeon interne qui pénètre dans la masse plasmique centrale et paraît constituer, sur une coupe, un îlot cellulaire entouré par le plasma à otolithes (fig. 3 et $4, i$. cel.)

La paroi de l'otocyste de l'Alciope adulte comprend, comme 
chez la larve, deux couches de cellules: l'une, répondant à l'hypoderme proprement dit; l'autre, aux éléments sensoriels de l'organe. Ces deux couches n'ont pas la même importance relative dans toutes les parties de la vésicule auditive. Ce sont les régions antérieure et médiane de cette vésicule dont la constitution histologique rappelle le plus celle que l'on observe chez les jeunes Asterope candida. Dans la figure 3, qui reproduit une coupe passant par la région médiane, les deux couches cellulaires de la paroi de l'otocyste sont bien visibles. La couche externe répondant à l'hypoderme est peu développée en comparaison de la couche interne. Elle est limitée extérieurement par une cuticule qui en dérive, et elle est parsemée de nombreux noyaux de forme plutôt sphérique; ces noyaux sont refoulés contre la cuticule et paraissent plus abondants le long du bord proximal que le long du bord distal de la vésicule auditive.

Le nombre des cellules de la couche externe augmente notablement durant l'évolution embryonnaire de cette vésicule. Comme le montre la figure 3 , les cellules répondant à l'hypoderme ne sont pas nettement séparées de la couche interne formée par les éléments sensoriels. A un fort grossissement, on distingue cependant, entre ces deux couches, une mince bande protoplasmique réticulée et fibrillaire qui dépend pour une part de chacune d'elles.

Les cellules sensorielles bordent la cavité centrale de l'otocyste. Elles sont allongées et disposées radiairement par rapport à cette cavité. Leurs noyaux, beaucoup moins allongés que pendant la période larvaire, sont ovoïdes. Ils ne sont plus ponctués de granulations chromophiles, mais contiennent d'ordinaire un seul nucléole soutenu par un léger réticulum nucléaire. Ces noyaux sont plutôt disposés vers l'extrémité périphérique de la cellule (fig. 5, ce. au.).

Les éléments sensoriels possèdent un protoplasma finement granuleux qui se condense, à l'extrémité centrale de ces élé- 
ments, en une mince lame d'apparence cuticulaire. Celle-ci est très irrégulière; elle se découpe en dentelures plus ou moins accusées qui s'enfoncent dans la masse plasmique tenant en suspension les otolithes (fig. 5, de.). En examinant attentivement mes coupes, il m'a paru que la lame dentelée était encore traversée par de fins prolongements protoplasmiques ressemblant à des cils, et établissant une relation plus intime entre les cellules sensorielles et la masse plasmique centrale. Les extrémités périphériques de ces cellules se terminent par des fibres qui pénètrent dans la zone fibrillaire intercalée entre les deux couches sensorielle et hypodermique, et la constituent en partie (fig. 5, fi.). Ces fibres se mettent en rapport avec les ramifications du nerf auditif, et n'en sont sans doute que les prolongements.

Les figures 3 et 4 montrent un léger dépôt pigmentaire qui s'est effectué dans la portion dorsale de l'otocyste. Ce dépôt ne commence à se produire que relativement tard puisqu'il n'est pas encore visible chez les larves d'Alciopes déjà pélagiques. Dans l'espèce qui nous occupe, quoique la couche des éléments sensoriels entoure complétement la masse plasmique centrale à otolithes, on observe que ces cellules auditives sont plus serrées et montrent une disposition radiaire plus accusée, le long de la face interne on proximale de l'otocyste que le long de la face externe ou distale de ce dernier.

La figure 4 représente une coupe passant par la région postérieure de l'organe auditif, région à laquelle correspond le pédicule qui rattache cet organe au corps de l'Alciope. La masse plasmique centrale a diminué de volume; elle est refoulée en partie par un de ces îlots cellulaires dont j'ai parlé précédemment, et qui constituent des bourgeons de la paroi de l'otocyste. Les contours de cette masse plasmique ont été un peu schématisés dans la figure 4, pour ne pas trop surcharger le dessin. Ils sont assez irréguliers car il s'y développe de petites expansions 
qui pénètrent dans la couche des cellules sensorielles. Ces dernières conservent les mêmes caractères histologiques que sur la coupe représentée aux figures 3 et 5 , coupe que je viens de décrire en détail. Je n'en parlerai donc pas; je ne ferai que mentionner l'existence d'un petit amas de noyaux situé en dehors de la couche des éléments sensoriels, au point d'origine du court pédicule de la vésicule auditive (fig. $4, g$. au.). Ces noyaux sont sphériques et leur hyaloplasme est parsemé de petites granulations chromophiles; ils sont plongés dans un protoplasma finement granuleux et fibrillaire. L'amas cellulaire en question se met en rapport avec l'extrémité périphérique des cellules sensorielles et il s'en détache des fibrilles qui se dirigent vers le ganglion sous-œsophagien. Ce ne peut être qu'un ganglion auquel je donnerai le nom de ganglion acoustique.

La couche cellulaire externe de la paroi de la vésicule auditive, celle qui répond à l'hypoderme prend dans cette région postérieure de la vésicule une plus grande importance. Dans la. figure 4, cette couche est surtout développée le long de la face proximale ou face de fixation de l'organe. J'y ai constaté des fibres musculaires localisées surtout à la face ventrale de cette région et qui me paraissent être en voie de rétrogradation. Nous nous trouvons certainement en présence d'un faisceau musculaire rudimentaire.

Le dépôt pigmentaire, signalé en étudiant la coupe représentée à la figure 3 , se retrouve à la face dorsale de la région postérieure de l'otocyste. Il pénètre non seulement dans la couche hypodermique, mais aussi dans la couche sensorielle; il a une colaration brunâtre et se résoud en très fins granules pigmentés.

J'ai constaté chez l'Astérope un fait au premier abord paradoxal. Cette Annélide possède non pas deux, mais quatre sacs auditifs distribués par paires. La première paire est, comme nous l'avons vu, appendiculée au premier segment troncal et inner- 
vée par le ganglion sous-œsophagien; la seconde paire est appendiculée au second segment troncal et innervée par le second ganglion de la chaîne nerveuse ventrale. C'es organes sont donc métamériques. La seconde paire d'otocystes possède la même structure histologique et les mêmes caractères morphologiques que la première; son développement est seulement plus tardif, puisque dans la larve pélagique étudiée au commencement de ce travail, les otocystes antérieurs étaient déjà nettement accusés alors que la paire postérieure de ces organes était représentée par de simples bourgeons ectodermiques. Suivant les espèces considérées, il doit se produire des variations dans la précocité relative d'apparition des deux paires de vésicules auditives, car celles-ci sont inégalement développées chez les différentes formes d'Alciopides. Peut être certaines espèces de ces Annélides ne possèdent-elles qu'une paire de ces organes, alors que d'autres en ont plus de deux paires.

Puisque les sacs auditifs des Astéropes représentent des appendices du corps et ont une disposition métamérique, il importait de savoir s'ils étaient encore répartis sur d'autres segments troncaux, ou bien, s'il existait sur ces segments des organes qu'on pût leur homologuer. J'ai fait plusieurs séries de coupes en différentes régions du corps et n'ai pas trouvé d'autres otocystes que les deux paires post-céphaliques. J'ai constaté cependant, que chaque segment de l'Astérope porte une paire d'organes qui ont la même valeur morphologique que les vésicules auditives et sont par conséquent leurs homologues.

Pour bien saisir cette homologie, il est nécessaire de se reporter à la constitution des organes locomoteurs types des Annélides Polychètes. Les pieds de ces Vers constituent sur les faces

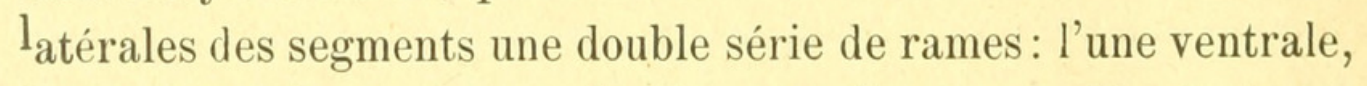
l'autre dorsale, accompagnée chacune d'un appendice tentaculiforme ou cirre. Les Alciopides, elles, ne possèdent qu'une rangée d'organes locomoteurs correspondant aux parapodes ven- 
traux. Ces organes sont soutenus par un acicule et portent un faisceau de soies tantôt simples, tantôt composées. Ils sont flanqués d'un cirre ventral se présentant sous forme d'une expansion lamelleuse, foliacée des parapodes, disposition éminemment favorable à la natation puisqu'elle augmente la surface de propulsion du pied.

On observe, chez les Alciopides, une seconde expansion foliacée annexée à la face dorsale du parapode et correspondant à un cirre dorsal. Ce dernier a une individualité plus accusée que celle du cirre ventral. Il s'insère généralement par un court pédoncule sur la portion basilaire du parapode, mais, dans la région antérieure du corps, il tend à se détacher du pied et à former un appendice indépendant. Dans la grande majorité des segments de l'Alciopide, les cirres dorsaux fonctionnent comme organes locomoteurs grâce à leur disposition foliacée; par contre, dans les segments post-céphaliques, ces cirres deviennent coniques ou piriformes et jouent le rôle d'organes sensoriels. C'est alors qu'ils s'isolent davantage des parapodes adjacents lesquels diminuent d'importance et se réduisent plus ou moins.

En étudiant la région antérieure du corps d'une Astérope, on reconnaît sans peine que les deux paires de sacs auditifs de ces Alciopides correspondent aux cirres dorsaux foliacés des segments postérieurs. Ainsi les otocystes de ces Annélides ne sont que des cirres parapodiaux transformés.

Les appendices auditifs post-céphaliques des Alciopes sont trop apparents pour n'avoir pas déjà attiré l'attention des observateurs. Claparède ${ }^{1}$ et d'autres auteurs les ont signalés et figurés, mais les ont considérés à tort comme de simples cirres tentaculaires. CARUS ${ }^{2}$ dit entre autres, dans sa diagnose de la famille des Alciopea : lobus cephalicus distinctus..., segmenta post

${ }^{1}$ Claparède, Les Annélides Chétopodes du golfe de Naples. Mém. Soc. Phys. et d'Hist. nat. Genève, 1868-1870.

${ }^{2}$ Carus, Prodromus Faunae Mediterraneae. Stuttgart, 1884. 
lobum cephalicum cirris tentacularibus nec pinnis..., etc.. Ce dernier caractère n'est pas exact. Les cirres dorsaux qui se sont différenciés en organes auditifs dans la région post-céphalique, sont très volumineux et paraissent former à eux seuls les appendices de ces segments. Il n'en est rien, car les parapodes de cette région ne se sont pas complètement atrophiés. Ils se retrouvent sous forme de petits bourgeons ectodermiques portant un faisceau de soies encore visible, comme on peut s'en assurer en examinant la coupe représentée à la figure $4(p a . v$.).

GREEFF ' a aussi observé et figuré les appendices vésiculeux post-céphaliques des Alciopides, seulement au lieu de leur attribuer des fonctions sensorielles il en fait des réservoirs ou sacs spermatiques. Il dit dans sa description de l'Alciope longirhyncha: "Sehr bemerkenswerth ist das erste auf den Kopf « folgende Fühlercirrenpaar, das in meinem Exemplar in, auf * kurzen breiten Stielen sitzende, kugelförmige Organe um* gewandelt war (Fig. 37,e), die ganz mit dunkelkörnigen und * lebhaft sich bewegenden Körperchen erfüllt waren. Ich " glaube wohl nicht zu irren, wenn ich die letzteren als Sper" matozoiden deute, die entweder hier, und in diesem Falle in * den nach Lage und Form eigenthümlichen Hoden gebildet «sind, oder aus der Körperhöhle, vielleicht in Verbindung mit «segmentalorganen, aufgenommen und von hier zur Befruch« tung übertragen werden... Im Anschluss an die bei der obigen * Alciopa longirhyncha vorkommenden, zu Hoden oder Samen« behältern umgestalteten Fühlercirren, möge hier noch die « Beobachtung einer andern in gewisser Richtung analogen Er«scheinung Erwähnung finden. Sie betrifft ebenfalls eine * Rhynchonerella-Art, die im Uebrigen nicht viel Bemerkens* werthes zeigte, deren zweites und drittes Fussstummelpaar « einen überzähligen, zwischen Rückencirrus und Ruder liegen-

${ }^{1}$ Greeff, Ueber die pelagische Fauna an den Küsten der. Guinea-Inseln. Zeits. für wiss. Zool. t. 42, 1885 . 
" den blattförmigen Cirrus besassen, die alle dunkle rundliche « Körperchen enthielten, bald in einzelnen mehr oder minder « abgegrenzten rundlichen Feldern, bald in grösserer Ausdeh«nung, so dass die fraglichen Organe strotzend mit jenen Kör«perchen erfüllt waren. Bei genauerer Prüfung und Isolirung « der Körperchen stellten sich dieselben als spermatozoiden« ähnliche Gebilde dar, bestehend aus einem länglichen nach « hinten zugespitzten Vordertheil oder Kopf und einem faden« förmigen Anhang. »

Dans la description qui précède, GREEFF identifie des organes qui n'ont pas la même valeur morphologique. Je n'ai pas eu à ma disposition les espèces d'Alciopides dont parle ce savant; je ne puis par conséquent contrôler ses observations de visu et je dois, pour les discuter, m'appuyer exclusivement sur le texte et les dessins de cet auteur. Si je compare l'Alciope longirhyncha, que GREEFF représente à la figure 37 de son mémoire précité, à mes exemplaires d'Asterope candida, le doute n'est pas possible. Il ressort avec évidence de cette comparaison que les soidisant sacs spermatiques de l'Alciope sont identiques aux appendices auditifs de l'Asterope. Ils ont même forme, même position et même origine puisqu'ils correspondent aussi à des cirres dorsaux modifiés. Malheureusement GREEFF n'a pas étudié la structure histologique de ces organes; il a observé à l'intérieur de ces derniers de nombreux corpuscules, dont il n'a pas déterminé la nature, et se base sur la présence de ces corpuscules pour assimiler les appendices vésiculeux post-céphaliques de l'Alciope longirhyncha à des réservoirs spermatiques. Cette méprise s'explique en partie par le fait que GREEFF croyait avoir antérieurement découvert des vésicules auditives chez les Alciopides et considérait comme telles de volumineuses glandes unicellulaires annexées au globe oculaire de ces Annélides.

La citation du mémoire de GREEFF reproduite plus haut montre que ce savant cherche à justifier sa manière de voir, en 
s'appuyant sur des observations plus détaillées faites sur une espèce de Rhynchonerella. Cette espèce possède des expansions foliacées en relation avec les deuxième et troisième paires de parapodes, expansions qui renferment des corpuscules semblables à des spermatozoïdes (voir fig. 38-41 du travail de GreefF). Chez les femelles, ces expansions foliacées existent aussi, mais elles sont remplies par des œufs à divers états de développement. Je n'ai pas observé d'organes semblables dans les genres Asterope et Alciope. En tous cas, les sacs spermatiques ou ovulaires des Rhynchonerella ne peuvent être identifiés aux appendices auditifs de l'Alciope longichyncha et de l'Asterope candida; ils ne constituent pas des cirres dorsaux modifiés, mais représentent des organes surnuméraires qui de l'aveu même de GreEFF sont situés entre le cirre dorsal et le parapode.

Ce savant a-t-il commis une erreur d'observation en prenant pour des spermatozoïdes les corpuscules contenus dans les expansions foliacées de cette Rhynchonerella? S'est-il trompé en affirmant que ces expansions limitées à la deuxième et à la troisième paires de parapodes sont des cirres surnuméraires et non les cirres dorsaux eux-mêmes? Je l'ignore, ne possédant, dans mon matériel de travail, aucune espèce de ce genre. Il me parait cependant difficile de concilier l'existence de ces réservoirs spermatiques post-céphaliques avec la présence de tubes (organes segmentaires) remplis de spermatozoïdes, répondant selon GreefF aux $10^{\mathrm{me}}, 11^{\mathrm{me}}, 12^{\mathrm{me}}$ et $13^{\mathrm{me}}$ segments du Rhynchonerella fulgens. Ces tubes viennent déboucher à la face ventrale du corps dans des bourgeons dermiques spéciaux et me paraissent être les véritables réservoirs spermatiques de ces Alciopides (voir GREeFF op. cit. page 451 et ApsTEIN ${ }^{1}$ page 136).

Il résulte de ce qui précède que les appendices vésiculeux des

${ }^{1}$ Apstern, Die Alciopiden des Naturhistorischen Museums in Hamburg. Jahrb. wiss. Anst. Hambourg, 8me année, 1890. 
premiers segments troncaux des Alciopides ont été pris soit pour de simples cirres tentaculaires, soit pour des sacs spermatiques. J'ai montré que, dans la région post-céphalique, les cirres dorsaux des parapodes se différencient les uns en tentacules, les autres en otocystes, ce qui explique la position métamérique de ces derniers. Quoique ces otocystes soient d'ordinaire annexés aux deux premiers segments troncaux, il est fort possible - étant donné leur origine - qu'ils se développent aussi sur des métamères plus postérieurs. Il me paraît probable que, dans la Vanadis fasciata décrite par APSTEIN ', les cirres dorsaux des sixième et septième segments représentent des organes auditifs.

Peut-être aussi ces otocystes parapodiaux ne se rencontrentils pas chez toutes les espèces d'Alciopides. J'ai constaté, par exemple, d'intéressantes différences dans le degré de développement de ces organes, en comparant les deux genres Asterope et Alciope. L'Alciope contrainii est une forme très instructive à cet égard, car elle nous permet d'étudier une des principales étapes par lesquelles le cirre dorsal a passé pour se transformer en un appendice auditif. La figure 7 représente une coupe d'un otocyste de cette Alciope. Elle nous montre que dans la région distale de l'organe, la couche hypodermique s'invagine en un double repli dans les concavités duquel sont contenus les grains chromophiles ou otolithes. Les cellules de ce double repli s'allongent, deviennent sensorielles (fig. $7, c e . s$.), tandis que dans les régions latérales et proximale de l'otocyste, la couche hypodermique a conservé sa structure histologique habituelle (fig. 7, hy.). L'intérieur de l'appendice auditif est rempli par une masse protoplasmique plurinucléée identique à celle que l'on observe dans la partie centrale du parapode (fig. $7, m . p . p$.).

Les appendices auditifs de l'Alciope contrainii ont donc

1 Apstein, Vanadis fasciata, eine neue Alciopide. Zool. Jahrb. Syst. Abth., t. อ.. 1891.

Rev. Suisse de Zool., T. I. 1893. 
gardé, dans leur organisation, des preuves indéniables de leur origine parapodiale et chez cette espèce, la transformation des cirres dorsaux en otocytes n'est en somme qu'ébauchée. Supposons que le processus d'invagination de la couche hypodermique s'accuse davantage, il se formera une vésicule auditive à double paroi: l'une externe, protectrice; l'autre interne, sensorielle. Nous obtiendrons ainsi une disposition semblable à celle que j'ai décrite chez Asterope candida, espèce chez laquelle les otocystes me paraissent avoir atteint leur plus grande différenciation.

Les organes auditifs que possèdent sinon toutes, du moins un certain nombre d'espèces d'Alciopides, montrent d'intéressantes particularités et s'éloignent complètement, au premier abord, du type des otocystes décrit chez d'autres formes animales. Leurs otolithes sont des dérivés nucléaires, leur paroi se décompose en deux couches cellulaires: l'une externe hypodermique, indifférente; l'autre interne sensorielle, dérivant aussi de l'hypoderme. Ces organes dépendent des premiers segments troncaux et ont une disposition métamérique. Enfin, ils représentent des cirres dorsaux modifiés et ont une origine parapodiale manifeste ainsi que le prouve leur étude morphologique et embryogénique.

Les otocystes apparaissent relativement tard dans l'ontogenèse des Alciopides; du moins, les cirres dorsaux aux dépens desquels ils se différencient, ne prennent les caractères de vésicules auditives que dans les stades larvaires assez avancés. En suivant l'évolution des organes parapodiaux dans différents stades compris entre $0^{\mathrm{mm}}, 5$ et $3^{\mathrm{mm}}$, on voit les parapodes proprement dits se former en premier lieu. Ceux-ci appartiennent dès l'abord à la face ventrale des segments et, pendant la croissance de la larve, paraissent se reporter vers la ligne médio-latérale du corps.

Après les parapodes, se montrent les cirres dorsaux qui ne naissent pas sur les parapodes eux-mêmes, mais se forment un 
peu dorsalement par rapport à ces derniers. Durant leur développement, les bourgeons parapodiaux font toujours plus saillie sur les côtés des segments; leur région basilaire s'allongeant davantage, entraîne avec elle les cirres dorsaux adjacents qui, dans l'adulte, font partie intégrante du pied des Alciopides. Les cirres dorsaux comprennent un pédoncule proximal et une extrémité distale renflée qui ne tarde pas à devenir foliacée. Dans les segments post-céphaliques, l'extrémité distale de ces organes se renfle en une sphère ectodermique (fig. 8, va au.) réprésentant la première ébauche de la vésicule auditive de l'adulte. Tôt après le cirre dorsal, se dessine le cirre ventral dont l'évolution est ainsi plus tardive et dont les relations avec le parapode sont plus étroites.

Pour être complet, il me reste à mentionner les glandes segmentaires qui se présentent sous forme de petites protubérances en connexion avec les bourgeons parapodiaux. Ces protubérances commencent à devenir visibles chez des larves de $3^{\mathrm{mm}}$; elles sont annexées à la face dorsale des segments et prennent une extension plus ou moins grande suivant les espèces. Généralement, il existe chez les Alciopides une paire de glandes segmentaires par métamère; la Greffia celox cependant en possède deux paires par segment, l'une dorsale, l'autre ventrale. La figure 6 représente une coupe d'un de ces organes appartenant à l'Asterope candida. La glande dessine, à la face dorsale du corps de cette Alciopide, une protubérance creuse dont la cavité est remplie d'un protoplasma multinucléé, granuleux, identique à celui qui est contenu dans la portion basilaire du parapode (fig. $6, m . p . p$.). La paroi de la protubérance est formée par une épaisse couche hypodermique constituée surtout par des cellules glandulaires, entre lesquelles sont intercalées des cellules allongées, probablement sensorielles. Les éléments glandulaires sécrètent des granulations pigmentaires brunâtres qui sont distribuées plus régulièrement à la périphérie que dans 
la région profonde de la couche hypodermique (fig. $6, p i$.). Les glandes segmentaires jouent sans doute à la fois un rôle glandulaire et sensoriel. Peut être contribuent-elles à renseigner les Alciopides sur les variations thermiques qui se produisent dans le milieu ambiant, variations qu'il est utile à un animal pélagique de pouvoir enregistrer. Ces protubérances pigmentées dorsales s'atrophient dans les premiers segments post-céphaliques, du moins chez Asterope candida et Alciope contrainii; les appendices auditifs peuvent alors partiellement se charger de pigment (fig. 3 et $4, p i$.).

L'origine parapodiale des otocystes constitue-t-elle un fait morphologique général chez les Annélides, ou se rencontre-t-elle chez les Alciopides seulement? Les données anatomiques et embryogéniques que nous possédons sur les organes auditifs des Polychètes sont encore trop imcomplètes pour répondre d'une manière satisfaisante à cette question. Les otocystes des Arénicoles ont été fort bien décrits, soit par JouRdaN dont j’ai déjà analysé la communication au commencement de mon travail, soit surtout dernièrement, par EHLERs'. Ils fournissent un intéressant terme de comparaison avec les vésicules auditives des Alciopides. Ehlers a étudié les otocystes de quatre espèces d'Arenicola et a trouvé que la constitution de ces organes varie d'une espèce à l'autre. C'est chez l'Arenicola Claparedii qu'ils sont le plus rudimentaire. Ils consistent en une simple vésicule communiquant encore avec l'extérieur par un orifice et dont la paroi n'est pas différenciée en un épithélium sensitif; les otolithes font défaut. Chez Arenicola marina, les vésicules auditives sont un peu mieux caractérisées. Elles sont en relation avec le système nerveux central; des granulations minérales provenant de l'extérieur y pénètrent et fonctionnent comme des otolithes. Chez l'Arenicola Grubii et l'Arenicola antillensis les

${ }^{1}$ Ehlers, Die Gehörgane der Arenicolen. Zeit. wiss. Zool. t, こ̌3, 1892. 
otocystes atteignent leur maximum de différenciation. Ils sont clos, pourvus d'un épithelium sensitif et se rattachent par un nerf à l'anneau nerveux périœsophagien. Les otolithes qui flottent dans la cavité de ces organes sont des produits particuliers de ceux-ci. Dans ces deux dernières espèces d'Arénicoles, les vésicules auditives sont enfoncées plus ou moins profondément dans la couche musculaire de la paroi du corps, et appartiennent plutôt à la face dorsale de l'animal.

Les quatre formes d'Arénicoles étudiées par EHLERs nous montrent que les organes auditifs de ces Polychètes passent de l'état d'une simple cavité ou otocrypte, encore ouverte à l'extérieur et sans otolithes propres, à l'état d'une vésicule auditive typique ou otocyste. Selon EHLERs, les otocystes dérivent phylogénétiquement des otocryptes, et chez toutes les espèces animales qui en possèdent, les organes auditifs apparaissent pendant l'évolution embryonnaire, sous forme d'invaginations ectodermiques rappelant les otocryptes primitifs. Ce savant en conclut, mais d'une manière dubitative, que des quatre Arénicoles mentionnés plus haut, l'Arenicola Claparedii seule a encore conservé la disposition ancestrale de la vésicule auditive de ces Annélides; puisqu'elle possède des otocryptes.

L'argumentation de EHLERs a une grande valeur, cependant elle a le tort de supposer une unité du mode de formation des otocystes dans la série animale. Certes, ces organes prennent toujours naissance aux dépens de l'ectoderme, mais ne représentent-ils que de simples différenciations d'une même ébauche ancestrale? C'est ce que je ne crois pas. Sans sortir des Annélides, il me paraît impossible de faire dériver les appendices auditifs des Alciopides dout l'origine parapodiale est incontestable, des otocystes dont sont pourvus certaines larves Trochophores de ces Vers.

Les vésicules auditives de ces larves sont probablement primaires, car elles se rencontrent non seulement dans la 
Trochophore des Annélides mais aussi dans la Trochophore des Mollusques. On les a même observées dans la larve ancestrale type des Trochozoaires ', celle des Archiannélides. FraIPONT dit à ce sujet, en décrivant la plaque syncipitale d'une Trochophore $^{2}$ de Polygordius de la $3^{e}$ période: "Sur une coupe optique plus profonde la disposition des cellules en ganglions distincts se marque clairement. Ils (ces ganglions) ont la forme de deux reins ou haricots dont le hile est tourné vers l'intérieur. Ils contiennent chacun en arrière un organe formé par cinq à six cellules claires délimitant une petite cavité. Ce sont probablement là des otocystes ou organes auditifs (?) » (Op. cit. page 68). J'ai eu l'occasion de vérifier cette observation de FraIPONT et je ne puis que la confirmer. La Trochophore des Polygordius est pourvue de vésicules auditives et cependant les Polygordius sont des Annélides si primitives que chez elles les parapodes ne se sont pas encore développés.

Les otocystes des Alciopides sont certainement secondaires puisqu'ils résultent de la transformation d'organes remplissant tout d'abord d'autres fonctions. Les cirres dorsaux de certains segments sont devenus par la suite des appendices auditifs chez ces Polychètes, de sorte que les otocystes des Alciopides dont l'origine est parapodiale n'ont pas la même valeur morphologique que ceux de la Trochophore d'autres Annélides quoiqu'ils remplissent un rôle physiologique identique.

Des organes qui se sont adaptés secondairement à des fonctions auditives, peuvent subir un développement rétrograde pendant la phylogenèse de l'espèce et, par une sorte de convergence, revêtir des caractères rappelant ceux des otocystes primaires. Supposons par exemple que les appendices auditifs

${ }^{1}$ Le groupe des Trochozoaires, introduit, si je ne me trompe, par Roule dans la science, renferme les Archiannélides, les Annélides y compris les Molluscoïdes et les Mollusques.

${ }^{2}$ Fraipont, Le genre Polygordius. XIVe monographie de la Fauna und Flora des Golfes von Neapel, 1887. 
des Astéropes se réduisent peu à peu et finissent par s'enfoncer dans la couche dermo-musculaire du segment. Ils se constituera ainsi des vésicules ouvertes, dont la couche sensorielle de la paroi pourra plus ou moins s'atrophier, et qui paraîtront des formations primitives, ancestrales, alors qu'elles seront dues, en réalité, à un processus de dégénérescence. C'est à cette catégorie de formations que se rattachent, selon moi, les otocryptes de l'Arenicola Claparedii. Les otocryptes de cette dernière espèce ne constituent donc pas l'ébauche auditive aux dépens de laquelle les otocystes des autres Arénicoles se sont différenciés, mais elles représentent l'état le plus dégradé d'une série évolutive descendante dont les termes moyens sont donnés par les otocystes des Arenicola Grubii, antillensis et de l'Aricia acustica, et dont le point de départ doit être sans doute cherché dans les appendices auditifs parapodiaux des Alciopides.

Le bien fondé de ce point de vue ressort de l'étude comparative des faits, et ne se justifie pas seulement par des considérations théoriques. Chez tous les animaux possédant des organes de l'audition, soit otocryptes, soit otocystes, les cellules ectodermiques qui constituent la paroi de ces organes acquièrent des caractères histologiques spéciaux, en rapport avec le rôle fonctionnel qu'elles ont à remplir. Elles donnent naissance à des prolongements protoplasmiques plus ou moins fins et mobiles qui font saillie dans la cavité des sacs auditifs; elles se mettent en relation avec le système nerveux central. Or, chez Arenicola Claparedii, les otocryptes ne montrent pas ces caractères fonctionnels particuliers que l'on peut déjà observer dans les vésicules auditives de la larve Trochophore des Annélides. D'après EHLERs, il n'existe pas d'épithélium sensitif dans les otocryptes de cette Arénicole et le nerf acoustique fait défaut. Cette absence de caractères fonctionnels est l'indice évident d'une dégradation, d'une dégénérescence qui commence à se manifester dans les organes auditifs de l'Arenicola marina, et s'accentue surtout dans ceux de l'Arenicola Claparedii. 
Afin de mieux comprendre la morphologie des otocystes des Arénicoles, il faudrait connaître leur ontogenèse, et celle-ci nous est encore inconnue. Aussi, pour déterminer comment ces organes se sont constitués dans la phylogenèse de ces Polychètes, nous en sommes réduits à baser nos déductions sur le mode de distribution et de position de ces otocystes par rapport aux segments du corps et aux appendices qui en dépendent. Si ce mode de distribution et de position correspond à celui que j'ai signalé chez les Alciopides, nous pourrons en conclure avec beaucoup de probabilité que les vésicules auditives de ces familles de Polychètes ont la même origine.

SPENGEL ${ }^{1}$ a émis l'hypothèse que les otocystes des Arénicoles dérivent des organes de la nuque, en allemand Nackenorgane, qui sont assez répandus chez les Annélides surtout pendant la période larvaire. Ces organes de la nuque sont de petites fossettes creusées dans le segment céphalique et tapissées par des cellules ectodermiques portant des cils vibratiles; ils sont innervés par les ganglions cérébroïdes. Les observations anatomiques plus récentes ne justifient pas l'hypothèse de SPENGEL. Comme Ehlers l'a montré. les Arénicoles possèdent à la fois des organes de la nuque et des vésicules auditives. Ces dernières ne proviennent donc pas des premières.

EHLERs dans son mémoire précité a discuté avec beaucoup de sagacité la question de la morphologie des organes auditifs des Annélides. Comme ces organes ont une disposition métamérique, entre autres chez Aricia acustica, ce savant pense qu'ils doivent dépendre des appendices pairs des segments. Les otocystes ne peuvent se déduire des bourgeons parapodiaux, car on observe sur le même métamère à côté d'eux, les deux rames parapodiales ventrale et dorsale. Ils dérivent probablement des cirres parapodiaux. Cette hypothèse dont EHLERs n'a

1 Spengel, Oligognathus Bonelliae. Mitth. Zool. Station zu Neapel, t. III, 1882. 
pu encore vérifier la justesse chez Aricia acustica, se justifie pleinement chez les Alciopides ainsi que je l'ai démontré dans le cours de ce travail, et c'est aux dépens des cirres dorsaux des parapodes que les vésicules auditives de ces Alciopides se sont différenciées. Il paraît en être de même chez les Arénicoles et les Aricies, étant donné la position plutôt dorsale des otocystes et la distribution segmentaire de ces derniers, du moins chez Aricia. Mais chez les Annélides, les cirres parapodianx acoustiques se sont réduits et par suite de cette régression les otocystes qu'ils représentent se sont un peu enfoncés dans l'épaisseur du tégument.

Nous trouvons un processus semblable de différenciation de cirres parapodiaux, accompagné d'une régression de ceux-ci, chez les Capitellides dont les organes latéraux, étudiés par EIsIG ${ }^{1}$, sont certainement des cirres modifiés. Si les déductions de EIsIG sont exactes en ce qui concerne l'origine parapodiale des organes latéraux, opinion que je crois fondée, ces organes seraient donc les homologues des appendices auditifs des Alciopides et probablement aussi des otocystes des Aricies et des Arénicoles. Nous voyons par là combien les cirres parapodiaux sont susceptibles de revêtir différentes formes morphologiques dans les diverses familles des Annélides. Ils deviennent ici un organe latéral, là un appendice foliacé locomoteur, ailleurs des vésicules auditives ou des branchies; toutes les transformations qu'ils subissent sont liées aux différentes conditions d'existence des Annélides, et ont pour effet d'assurer toujours mieux la survie de l'espèce, en étendant le champ de l'activité physiologique de celle-ci.

D'après ce qui précède, on observe chez les Polychètes au moins deux types d'otocystes, dont l'un est phylogénétiquement plus ancien que l'autre. Les formes ancestrales des Trocho-

' Eisig, Die Capitelliden. XVIe monographie de la Fauna und Flora des Golfes von Neapel, 1887. 
zoaires (Annélides et Mollusques) ont certainement possédé des vésicules auditives comparables à celles que l'on rencontre encore dans la larve Trochophore de ces animaux. Ces vésicules se sont maintenues dans le groupe de ces formes ancestrales dont les Mollusques sont dérivés, tandis qu'elles se sont généralement atrophiées durant l'évolution phylogénétique des Annélides. Chez ces dernières, les otocystes primitifs s'observent parfois encore pendant l'état larvaire, mais ne se retrouvent probablement plus dans l'individu adulte. Les organes auditifs que possèdent les Polychètes actuels ont une tout autre origine ; loin d'être des organes ancestraux, ils ont acquis très tardivement leur fonction auditive et résultent d'une adaptation secondaire d'appendices métamériques dépendant des parapodes. Les otocystes des Alciopides, qui se sont formés aux dépens des cirres parapodiaux dorsaux, ont encore conservé leurs caractères d'appendices segmentaires, tandis que ces caractères se sont profondément altérés dans les vésicules auditives des Aricies et des Arénicoles.

Nous savons, depuis les travaux de Delage ', que chez les Mollusques et les Crustacés, les otocystes remplissent une double fonction. Ces organes servent non seulement à l'audition, mais encore à régulariser la locomotion. Si on parvient à les détruire ou à les enlever, il se produit une désorientation locomotrice manifeste et permanente dans l'animal opéré. Je ne puis dire si les vésicules auditives des Annélides jouissent des mêmes propriétés fonctionnelles que celles des Mollusques et des Crustacés, car je n'ai pas eu l'occasion, jusqu'à présent, de faire les expériences nécessaires pour élucider cette délicate question de physiologie comparée.

EHLERs pense que les otocystes et otocryptes des Arénicoles représentent surtout des organes d'équilibration du corps, car

${ }^{1}$ Delage, Sur une fonction nouvelle des Otocystes. Arch. Zool. Expér. t. $V, 1887$. 
des sons produits dans le voisinage de ces Polychètes ne paraissent pas avoir été perçus par eux. Ce savant indique comment un organe doit être histologiquement constitué, pour être apte à percevoir des excitations auditives et il ajoute en parlant des Arénicoles: "Eine derartige Organisation geht den hier vor* liegenden Otocrypten und Otocysten völlig ab; als Apparate, * welche akustisch functioniren, sind sie daher nach ihrem ana" tomischen Bau, so lange nicht andere physiologische Erfah« rungen beigebracht werden, nicht zu bezeichnen. » (Op. cit. p. 258.)

Il n'existe pas, au point de vue histologique, de différences fondamentales entre les organes auditifs proprement dits et les organes d'équilibration locomotrice ou statique. Tous deux sont représentés par des vésicules ouvertes ou closes renfermant des otolithes et dont la face interne porte des cils plus ou moins nombreux et développés. Ces cils dépendent généralement de cellules sensorielles ectodermiques dans le protoplasme desquelles des fibres nerveuses viennent se terminer (Mollusques, Vers); mais, parfois, ils sont directement fixés sur la paroi cuticulaire de la vésicule et des fibres nerveuses y pénètrent (poils acoustiques des Crustacés Décapodes.) Malgré les différences structurales qui séparent les vésicules à otolithes des Décapodes de celles des Mollusques, les otocystes de ces deux groupes d'animaux servent, à la fois, à l'audition et à l'orientation locomotrice. Ces deux fonctions peuvent donc être localisées dans un même organe sans être pour cela indissolublement liées l'une à l'autre. En est-il de même chez les Annélides? Cela est probable. Si les expériences relatives à cette question physiologique sont presque impossibles à tenter sur les Arénicoles, elles sont plus facilement réalisables sur les Alciopides, dont les otocystes sont appendiculés aux segments post-céphaliques et doivent pouvoir être sectionnés sans trop de difficulté. J'espère être à même, au printemps prochain, de faire quelques recherches expérimentales à ce sujet. 
N'oublions pas que la perception auditive, de même que la perception visuelle, est susceptible de varier en qualité et en quantité dans la série animale. Des vibrations du milieu ambiant qui n'excitent plus l'épithélium sensitif de notre oreille peuvent très bien encore impressionner les vésicules auditives d'autres groupes d'animaux. Le fait que l'Arenicola marina ne répondait pas aux ondes sonores produites dans son voisinage immédiat, ne prouve pas que cette Annélide ne puisse percevoir d'excitations auditives. Il montre tout au plus, que l'espèce précitée a une insensibilité acoustique plus ou moins complète pour les diverses tonalités utilisées dans les expériences d'EHLERs. Peut-être, cette Arénicole percoit-elle, dans le milieu liquide qu'elle habite, des vibrations qui échappent totalement à notre oreille, et qui produisent sur l'épithélium sensitif de ses otocystes des excitations sinon identiques, du moins de même nature que celles recueillies par notre organe auditif.

La faculté d'orientation locomotrice n'est pas nécessairement liée à la présence d'une vésicule à otolithes et à épithélium sensitif. Chose curieuse, les otocystes ont été rencontrés jusqu'à présent chez les Polychètes sédentaires exclusivement (Arénicoles, Térébelles, Aricies, Serpuliens). Chez les Polychètes errantes dont l'orientation locomotrice doit être plus développée, ces organes manquent, et la fonction d'équilibration a donc son point de départ dans un autre dispositif anatomique. Les Alciopides sont les seules Polychètes errantes actuellement connues qui possèdent des otocystes, ainsi que cela a été établi dans le présent travail.

L’oreille des Vertébrés est un exemple intéressant de la séparation des fonctions d'audition et d'équilibration. On a cru, jusque dans ces dernières années, que l'organe auditif des Vertébrés était aussi le siège du sens de l'équilibre. Les expériences de Flourens, de Cyon, de Brown-Séquard, de Goltz et de quelques autres physiologistes tendaient à localiser ce sens de 
l'équilibre dans les canaux semi-circulaires. On admettait, sur la foi de ces expérimentateurs, que la section des canaux semi-circulaires entraînait des troubles dans la faculté d'équilibration de l'individu opéré. Une étude plus attentive de la question a montré que l'oreille des Vertébrés ne sert pas à l'équilibration, mais uniquement à l'audition. STEINER ${ }^{1}$ en particulier a prouvé par de nombreuses expériences, que l'on peut détruire les canaux semi-circulaires et même enlever l'organe auditif des Requins sans déterminer de troubles dans la faculté d'équilibration de ces derniers. Les Requins auxquels on a reséqué le sac auditif et ses annexes nagent avec la même facilité qu'auparavant; cette opération n'a donc nullement altéré leur faculté d'orientation locomotrice.

Au point de vue morphologique, comme au point de vue physiologique, l'oreille des Vertébrés n'est pas assimilable aux otocystes des Mollusques, des Crustacés et des Annélides. J'ai déjà démontré que les vésicules auditives des Annélides doivent leur origine à des adaptations secondaires et ont apparu tardivement dans la phylogenèse de ces Vers. Il en est de même pour les sacs auditifs des Vertébrés qui, d'après les recherches récentes, se sont différenciés aux dépens d'organes sensoriels appartenant à la ligne latérale.

Je renvoie le lecteur, pour la discussion détaillée de ce problème de morphologie, aux travaux de AYers ${ }^{2}$ et de MitroPHANOW ${ }^{3}$. Selon AYERS: "The detailed comparaison of the * anatomical characters of the surface sense organs (canal or"gans) of the existing Ichthyopsida and of the embryonic "stages of the Sauropsida and Mammalia as well, fully sustains

${ }^{1}$ Steiner, Ueber das Centralnervensystem und über die Halbcirkelformigen Canäle des Haifisches. Mitth. K. preuss. Akad. Wiss. Berlin, 1886.

2 Ayers, The Vertebrate Ear. Journal of Morphology. Vol. VI, May 1892.

- Mitrophanow, Etude embryogénique sur les Sélaciens. Arch. Zool. Expérim. 3 me série, no 2, 1893. 
« the conclusion that the ear is only a modified member of this " group of sense organs.... The above conclusion is rendered * certain by my discovery that in Elasmobranchs the structural " connection between the ear organs and the surface canal organs " is for a long time maintained after the ear has migrated to its " internal home, and in some forms may be said with truth to "persist during the life of the individual.» (Op. cit. pages 314-315.)

Eisig (op. cit. pag. 518 et suivantes) et d'autres zoologistes ont admis que les organes latéraux des Capitellides sont les homologues des organes de la ligne latérale des Vertébrés. Si ce point de vue est juste, les sacs auditifs des Vertébrés qui se sont différenciés aux dépens de cette ligne latérale, devraient être homologués aux organes latéraux des Capitellides et partant aux otocystes des Arénicoles et des Alciopides.

Je crois que la thèse soutenue par Eisig repose sur des bases scientifiques bien chancelantes. Il me semble que ce savant a trop facilement transformé des dispositions analogiques en homologies réelles. Les organes que l'on compare doivent, pour être homologues, ou bien avoir une même valeur morphologique ou bien naître d'une même ébauche embryonnaire. Il existe chez les Annélides et chez les Vertébrés des bourgeons sensoriels distribués sur les côtés du corps; s'ensuit-il que ces bourgeons soient homologues? évidemment non. Pour établir cette homologie, il faut non seulement s'appuyer sur un mode semblable de distribution, mais surtout démontrer que les bourgeons sensoriels latéraux des Vertébrés dérivent phylétiquement de ceux des Annélides. Malgré les vues ingénieuses de EIsig, cette dérivation me paraît contredite par les données embryogéniques actuelles.

A priori, il est peu probable que ces organes - dont la présence chez les Annélides est loin d'être constante - se soient transmis à travers toutes les modifications morphologiques qui 
ont accompagné l'évolution du phylum Vertébré, alors que les caractères fondamentaux de ce phylum: notochorde, tube nerveux dorsal, fentes branchiales n'existent pas encore dans les Annélides souches. Les bourgeons sensoriels de la ligne latérale, représentant ainsi la disposition la plus primitive que les Vertébrés aient hérité des Chétopodes, devraient apparaître très tôt dans l'ontogenèse des Poissons et se retrouver chez les formes plus ancestrales encore, l'Amphioxus par exemple. Il n'en est rien. L'Amphioxus possède bien des cellules sensorielles, mais pas de ligne latérale proprement dite. Chez les Poissons, les organes latéraux se forment en premier lieu dans la région céphalique, tandis que chez les Capitellides par exemple, cette région en est dépourvue.

On voit se développer chez les Ichthyopsidés et principalement chez les Sélaciens, un épaississement ectodermique qui s'étend sur les côtés de la tête. Cet épaississement sensoriel se divise en trois parties: une auditive, aux dépens de laquelle l'oreille des Vertébrés prendra naissance; une branchiale proprement dite, qui se différenciera plus tard en organes latéraux céphaliques et en organes branchiaux, ces derniers au nombre de six paires chez les Sélaciens; une postérieure ou troncale, qui s'allonge d'avant en arrière et se démembre peu à peu en les organes de la ligne latérale du corps. Ainsi, dans la région céphalique des Poissons, il existe deux séries de bourgeons sensoriels: les organes latéraux, et les organes branchiaux. Les premiers sont plus dorsaux que les seconds. Ces faits découverts par FroRIEP ' me semblent difficiles à interpréter dans l'hypothèse de Eisig.

Sans parler de l'homologie des plus hypothétiques, que Eisig cherche à établir entre les ganglions parapodiaux des Chétopodes et les ganglions spinaux des Vertébrés, la thèse de ce

${ }^{1}$ Froriep, Zur Entwickelungsgeschichte der Kopfnerven. Verhandl. Anatom. Gesellsch. 1891. 
savant se heurte, au point de vue morphologique, à une difficulté insurmontable. Les organes latéraux des Capitellides sont métamériques tandis que les recherches de Mitrophanow ont établi : "que les organes latéraux des Vertébrés ne prennent - nulle part naissance d'une manière métamérique et qu'à aucun "stade de développement ils n'ont une disposition métamérique "dans tout le corps. » (Op. cit. page 215.) L'ébauche primitive de ces organes est donc asegmentée, continue, et la disposition segmentaire que ceux-ci peuvent présenter par la suite, est purement secondaire.

D'après ce qui précède, les sacs auditifs des Vertébrés ne sont pas les homologues des otocystes des Annélides, otocystes qui représentent, de même que les organes latéraux des Capitellides, des cirres parapodiaux modifiés.

Nous arriverons à des conclusions identiques en comparant les vésicules auditives des Crustacés à celles des Chétopodes. Ces vésicules sont, chez les Décapodes, généralement annexées à l'article basilaire des antennules. Elles ont une structure histologique spéciale et sont caractérisées par la présence de poils auditifs dans lesquels pénètrent des filaments nerveux. Or, chez plusieurs Malacostracés, on observe soit sur les antennes, soit sur le corps, des poils sensoriels tout à fait semblables à ceux qui tapissent les otocystes des Décapodes. Il est donc probable que, durant l'évolution du phylum Crustacé, les organes auditifs se sont développés par concentration en certaines régions de l'organisme, de poils auditifs primitivement disséminés sur toute la surface du corps. Ce sont des organes spéciaux à cette classe d'Arthropodes, et dont la différenciation a été relativement tardive puisqu'ils font défaut aux différentes formes larvaires des Crustacés et ne se montrent que dans les groupes supérieurs de ces Arthropodes. Les rapports existant entre les otocystes des Crustacés et ceux des Annélides sont d'ordre exclusivement fonctionnel et n'impliquent aucune parenté morphologique. 
La conclusion générale qui se dégage de cette longue discussion, c'est que les organes auditifs des Métazoaires à symétrie bilatérale se différencient d'une manière indépendante dans chaque phylum et ne peuvent par conséquent se ramener à un seul type morphologique. L'oreille des Vertébrés est un produit secondaire des organes latéraux céphaliques de ce sousembranchement. Les vésicules auditives des Crustacés se forment par une localisation de poils sensoriels disséminés d'abord sur tout le tégument. Chez les Trochozoaires, nous avons trouvé des otocystes primitifs, c'est-à-dire appartenant déjà à la larve ancestrale Trochophore de ces Métazoaires, et des otocystes dérivant d'une adaptation secondaire. Les premiers se sont conservés chez les Mollusques jusque dans l'individu adulte. On peut les observer aussi dans la larve Trochophore de certaines Annélides, mais ils disparaissent durant l'évolution larvaire de ces dernières, et peuvent être remplacées dans l'adulte par des vésicules auditives secondaires qui dérivent de cirres parapodiaux. Ceci explique pourquoi les otocystes sont si rares chez les Annélides.

Malgré la diversité de leur origine morphologique, les otocystes des Métazoaires à symétrie bilatérale remplissent soit en même temps des fonctions d'audition et d'orientation locomotrice (Mollusques, Crustacés), soit des fonctions d'audition seulement (Vertébrés). Ils montrent donc une certaine unité d'activité physiologique, qui a imprimé aux otocystes de ces Métazoaires une certaine unité structurale. Quelle que soit leur origine, ces organes sont toujours représentés par des vésicules ouvertes ou closes renfermant des otolithes et tapissées généralement par un épithélium sensitif cilié, quelquefois par des poils auditifs (Crustacés). Les otolithes peuvent être inorganiques ou organiques; ce sont, ou bien des corpuscules provenant du monde extérieur, ou bien des corpuscules sécrétés par l'otocyste même. 
Ces différences ont fort peu d'importance et n'influent en rien sur l'activité fonctionnelle de l'organe.

Doit-on établir une démarcation tranchée entre les vésicules auditives proprement dites et les vésicules servant à l'orientation locomotrice? Non, car je ne crois pas qu'il y ait là un dualisme physiologique, mais seulement deux modes d'expression d'une même fonction fondamentale, celle de recueillir les vibrations produites dans le milieu ambiant. Toutes ces vibrations ne sont certainement pas perçues comme sons, il faut pour cela une certaine vitesse et une certaine amplitude d'oscillations; mais toutes contribuent à renseigner l'animal sur les variations mécaniques qui se manifestent autour de lui. Ces variations sont le mieux enregistrées par les cellules ectodermiques à cils sensoriels. Ces cils, qui font saillie dans le milieu extérieur, ressentent les moindres oscillations de ce milieu et les transmettent à l'organisme dont ils dépendent. En somme, l'aptitude physiologique de recueillir les vibrations ambiantes sonores ou autres ne constitue pas une fonction nouvelle, mais représente un cas particulier de la sensation du toucher. Primitivement, elle est localisée dans les mêmes éléments histologiques, les cellules à cils sensoriels.

En général, chez les animaux qui vivent fixés et dontles conditions d'existence sont par conséquent assez constantes, la sensibilité tactile par contact direct et la sensibilité tactile indirecte, transmise par vibrations du milieu ambiant, ne se différencient pas encore l'une de l'autre. Chez les animaux qui mènent une vie indépendante et dont les conditions d'existence sont éminemment variables, il importe que l'individu soit plus exactement renseigné sur la nature des excitations qu'il reçoit. Aussi, la sensibilité tactile directe, plus grossière et la sensibilité tactile indirecte, plus délicate, tendront-elles à se séparer l'une de l'autre.

Les Hydropolypes, les Scyphopolypes, par exemple, qui vivent 
fixés, ne possèdent que des cellules à cils sensoriels, disséminées sur toute la surface du corps et sur les tentacules où elles sont abondantes. Les Méduses qui leur correspondent, Hydroméduses et Scyphoméduses, mènent une vie indépendante, et chez elles, certains groupes de cellules tactiles se délimitent en organes spéciaux de sensibilité tactile indirecte, les vésicules auditives. Il est intéressant de constater que dans la plupart de ces Méduses, ces vésicules représentent des tentacules modifiés. Afin que ces organes puissent recueillir des vibrations de plus en plus imperceptibles, il s'accumule dans leur cavité interne des otolithes qui contribuent à exciter les cils ou poils dépendant des cellules sensorielles de leur paroi.

Si les otocystes montrent dans toute la série animale une certaine unité de structure, quelle que soit leur origine, cela tient à ce qu'ils se différencient aux dépens des éléments sensoriels les plus primitifs, les plus répandus et les plus constants : les cellules tactiles. Comme ces dernières sont disséminées sur la surface entière du corps de l'animal, les vésicules auditives pourront prendre naissance en différentes régions de celui-ci. Il va sans dire que ces vésicules tendront à se former là où les cellules tactiles sont le plus accumulées.

Afin de comprendre comment la fonction de l'orientation locomotrice est liée à la faculté qu'ont les otocystes d'être excités par les oscillations mécaniques du milieu ambiant, partons d'une donnée facile à interpréter. Irritons un peu vivement un tentacule d'une Actinie épanouie, les autres tentacules s'infléchiront aussitôt vers le point touché. L'excitation locale perçue par l'Actinie est donc transmise par voie réflexe aux autres tentacules et les mouvements de ces derniers seront coordonnés du côté de la région lésée. Les sensations tactiles, à elles seules, permettent à ces animaux de bien localiser les excitations reçues, et d'orienter leurs mouvements avec assez de précision et de rapidité. 
Supposons une Méduse possédant des otocystes distribués le long du bord de son ombrelle. Toutes les vibrations se produisant dans le milieu ambiant se répercuteront sur les cellales tactiles de cette Méduse et principalement sur celles qui constituent les vésicules à otolithes. Suivant la direction de ces vibrations, telle ou telle de ces vésicules sera plus particulièrement excitée et les sensations locales ainsi produites permettront à l'animal de reconnaître le sens de déplacement des oscillations qu'il perçoit. Comme la Méduse est libre, elle pourra réagir soit en se rapprochant soit en s'éloignant du point d'origine de ces oscillations, et il se développera entre les sensations fournies par les otocystes et les contractions musculaires de l'ombrelle, des relations de plus en plus étroites. Les otocystes deviendront peu à peu, par excellence, les organes d'orientation de la Méduse et la renseigneront sur la nature des phénomènes plus ou moins éloignés qui se passent autour d'elle.

Les considérations que je viens d'exposer ne s'appliquent pas seulement aux organes à otolithes des Métazoaires inférieurs, Méduses et Cténophores, mais aussi aux otocystes des Mollusques, des Crustacés et des Annélides. Chez ces Métazoaires à symétrie bilatérale, la faculté que possèdent les vésicules à otolithes d'enregistrer des vibrations sonores ou autres, favorise l'orientation dans le milieu ambiant, et les relations qui s'établissent entre l'activité fonctionnelle de ces organes et la coordination des mouvements du corps deviennent toujours plus accusées.

Jusqu'à présent, je n'ai considéré que la perception de vibrations mécaniques ayant leur cause en dehors de l'animal. Mais ce dernier peut généralement se mouvoir avec plus ou moins de rapidité. En se déplaçant, l'individu produit des ondulations du liquide dans lequel il se meut, et ces ondulations à leur tour excitent les vésicules à otolithes. Ainsi, la locomotion s'accompagne de sensations qui en sont la conséquence mécanique 
nécessaire, et qui sont perçues, en partie par la surface tactile entière du corps, en partie par les otocystes. Cet enregistrement plus ou moins réflexe du mouvement par les vésicules à otolithes complétant le rôle joué par ces dernières dans la faculté d'orientation de l'animal, explique les troubles moteurs si remarquables que l'on observe après l'ablation de ces vésicules.

Il résulte de cet exposé que l'équilibration ou l'orientation locomotrice de l'individu dans le milieu ambiant, n'est pas la conséquence directe de l'activité physiologique des otocystes, elle en est seulement une conséquence éloignée. Ce n'est pas en qualité d'organes percevant des vibrations que les otocystes remplissent des fonctions d'orientation, mais simplement en qualité d'organes sensoriels. Les vésicules à otolithes, en accentuant toujours davantage la sensibilité tactile indirecte, constituent pour les animaux qui les possèdent un précieux moyen d'exploration. Elles enregistrent les phénomènes qui se produisent autour de l'individu et, par les excitations qu'elles fournissent, ces vésicules permettent à celui-ci de réagir par des mouvements appropriés. Il se développe ainsi entre le centre nerveux sensitif auquel les otocystes sont rattachés et les centres moteurs, des rapports de dépendance de plus en plus marqués, rapports que l'on a improprement désignés sous le nom de sens de l'orientation locomotrice.

En somme, plus un organe sensoriel établit des relations étendues entre l'animal et le milieu extérieur, plus aussi il tiendra sous sa dépendance les mouvements du corps et jouera un rôle prépondérant dans le développement de la fonction physiologique complexe de l'équilibration et de l'orientation locomotrice. Chez bon nombre de Métazoaires, cette fonctıon est liée à la sensibilité tactile (la plupart des Vers, des Arthropodes, des Échinodermes); chez d'autres, elle est liée aux vésicules à otolithes (certains Hydroméduses, Mollusques, Crustacés Décapodes, quelques Annélides); chez certaines espèces enfin, elle 
dépend à la fois des organes visuels et des otocystes Crevettes, Mysis ${ }^{1}$, Scyphoméduses).

Chez les Vertébrés, la fonction d'équilibration du corps ne peut être rapportée à l'activité d'un seul organe sensoriel, elle se rattache d'un côté à la sensibilité générale, de l'autre à la sensibilité spéciaie et représente une résultante physiologique plus complexe encore que chez les Invertébrés. Il est probable que chez les Vertébrés aquatiques, les bourgeons tactiles de la ligne latérale contribuent à la fonction de l'orientation locomotrice. Les sacs auditifs de ces Métazoaires qui constituent, comme nous l'avons vu, une différenciation de la portion céphalique de cette ligne latérale, ne paraissent pas être en rapport avec cette fonction et servent exclusivement à percevoir les ondes sonores.

En résumé, les vésicules à otolithes sont des organes chargés de recueillir les vibrations produites dans le milieu ambiant. A l'origine, ces vibrations sont perçues comme sensations tactiles; puis, à mesure que ces vésicules se différencient davantage, elles deviennent excitables par des vibrations plus délicates, les ondes sonores. Le sens de l'orientation locomotrice n'est pas un sens spécial dépendant des otocystes; il représente une fonction physiologique d'ordre complexe à laquelle peuvent concourir isolément ou simultanément les principaux organes sensoriels de l'animal. L'ouïe n'est en dernière analyse qu'un toucher à distance; c'est pourquoi les vésicules à otocystes sont si souvent des dérivés d'appendices tactiles, tels que les tentacules des Méduses, les cirres parapodiaux des Annélides, les antennules des Crustacés Décapodes.

: Voir Delage, op. cit, page 25. 
c. $h y .=$ couche hypodermique.

c. sens. $=$ conche sensorielle.

c. $c .=$ cavité centrale de l'otocyste.

ce. $a u .=$ cellules acoustiques.

ce. $g l .=$ cellules glandulaires.

$d e=$ dentelures des cellules acoustiques.

$f . m$. = faisceaux musculaires.

$f i=$ fibres nerveuses des cellules acoustiques.

g. $a u .=$ ganglion auditif.

g.s. = ganglion sous-œsophagien .

$g r .=$ grains chromophiles ou otolithes. $h y .=$ hypoderme.

i. $c e l .=$ îlots cellulaires contenus dans la cavité de l'otocyste.

$m . p . p .=$ masse protoplasmique plurinucléée.

$n . a u .=$ nerf anditif.

pa. = parapode.

pi. = pigment.

$p r . d .=$ protubérances dorsales ou glandes segmentaires.

s. $=$ soies.

v. $a u .=$ otocyste.

Fig. 1. Coupe de l'otocyste appendiculé au premier segment troncal d'une larve pélagique d'Asterope candida. Cette coupe passe par le pédoncule de l'otocyste. Zeiss. apo. 16mm, oc. comp. 12. gross. $\times 187$.

Fig. 2. Coupe passant par la région équatoriale du même organe auditif et montrant une des glandes unicellulaires aux dépens desquelles se forment des otolithes. Zeiss. apo. $4^{\mathrm{mm}}$, oc. comp. 4, gross. $\times 250$.

Fig. 3. Région équatoriale d'un otocyste d'Asterope candida adulte. Zeiss. apo. $16 \mathrm{~mm}$, oc. comp. 4 , gross. $\times 62$.

Fig. 4. Région pédonculée de cet otocyste. Zeiss. apo. $16 \mathrm{~mm}$, oc. comp. 4, gross. $\times 62$.

Fig. 5. Portion de la couche sensorielle de ce même otocyste. Zeiss. apo. im. hom. $2 \mathrm{~mm}$, oc. comp. 4, gross. $\times \check{500}$.

Fig. 6. Parapode et glande segmentaire d'un segment de la région moyenne du corps d'un Asterope candida. Zeiss. apo. 16 $\mathrm{mm}$, oc. comp. 4, gross. $\times 62$.

Fig. 7. 'Coupe de l'otocyste d'un Alciope contrainii. Zeiss. id., gross. $\times 62$.

Fig. 8. Ebauche d'organe auditif d'une embryon d'Alciopide de $3 \mathrm{~mm}$ appartenant probablement à Asterope candida. Zeiss. apo. $4 \mathrm{~mm}$, oc. comp. 4. gross. $\times 2$ \%०

Toutes les figures ont été dessinées à la chambre claire. 
Rev. Suisse de Zool. T.I. 1893.

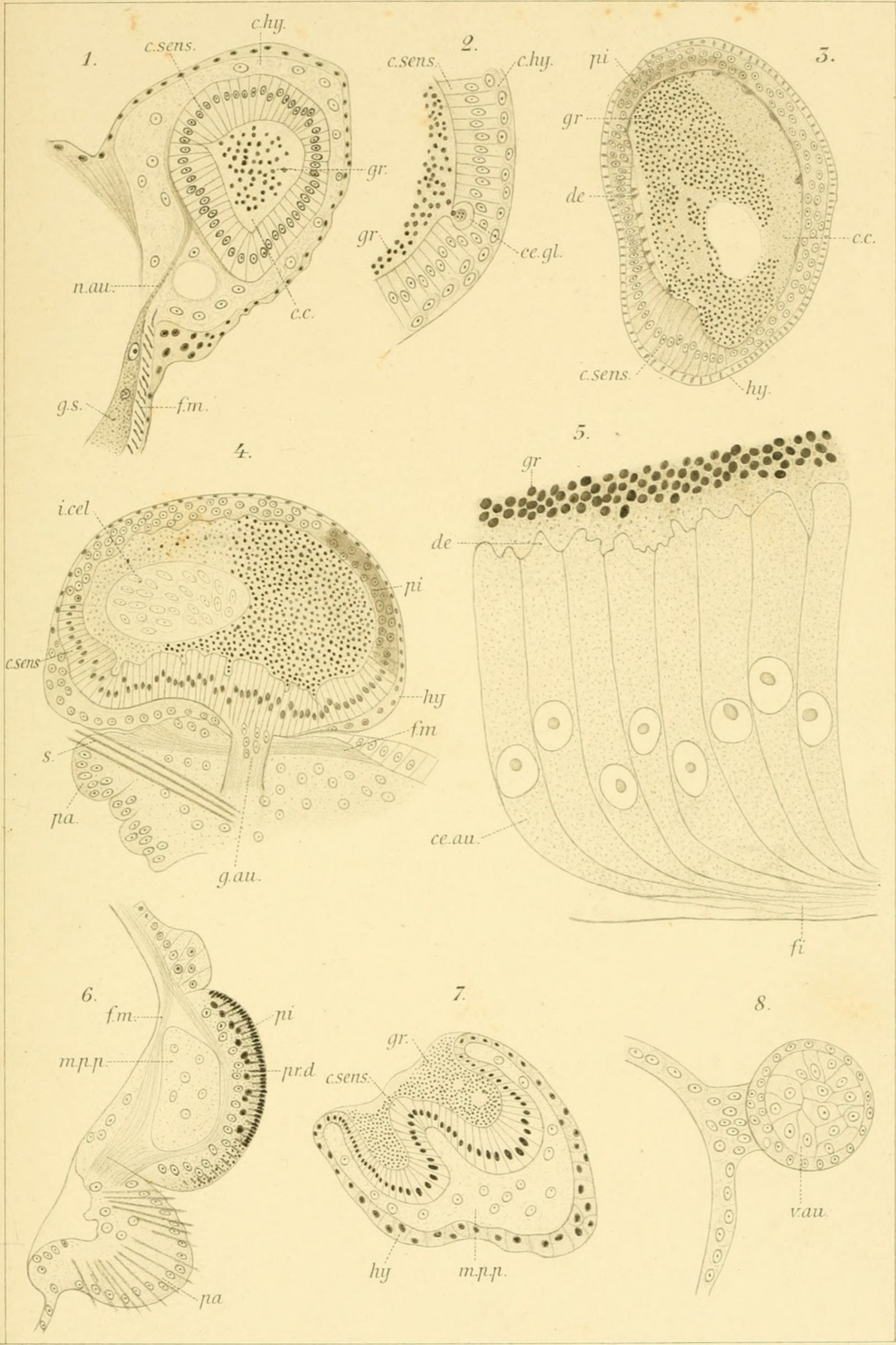

Ed.Béraneck. Alciopides. 


\section{$2 \mathrm{BHL}$ Biodiversity Heritage Library}

Béraneck, Ed. 1893. "L'organe auditif des Alciopides." Revue suisse de zoologie 1(3), 463-500. https://doi.org/10.5962/bhl.part.75132.

View This Item Online: https://www.biodiversitylibrary.org/item/38483

DOI: https://doi.org/10.5962/bhl.part.75132

Permalink: https://www.biodiversitylibrary.org/partpdf/75132

\section{Holding Institution}

MBLWHOI Library

\section{Sponsored by}

MBLWHOI Library

\section{Copyright \& Reuse}

Copyright Status: NOT_IN_COPYRIGHT

This document was created from content at the Biodiversity Heritage Library, the world's largest open access digital library for biodiversity literature and archives. Visit BHL at https://www.biodiversitylibrary.org. 